\title{
LETTER
}

\section{Three dimensional collagen scaffolds promote iPSC induction with higher pluripotency}

\section{Dear Editor,}

Extracellular environment plays a role in regulating stem cell fates and three dimensional (3D) scaffolds can be utilized to mimic the internal environment in vitro. Currently, many types of cells have been cultured in 3D conditions but only few studies have focused on reprogramming in a 3D environment. 3D culture systems provide circumstances that can better simulate native conditions which are comprised of distinctive cell morphology, oxygen levels, extracellular matrix secretion and concentration gradients of signaling factors (Keung et al., 2010; Gu et al., 2016).

Herein, we used collagen, the major composition of the extracellular matrix (Di Lullo et al., 2002), that serves as scaffolds to offer porous 3D surrounding to mimic in vivo environments (Song et al., 2015) and to explore the role of 3D conditions in reprogramming. In this study, we investigated the effect of $3 D$ collagen scaffolds on the reprogramming of mouse embryonic fibroblasts (MEFs) and pig embryonic fibroblasts (PEFs). MEFs could be successfully converted into mouse induced pluripotent stem cells (iPSCs) in 3D collagen scaffolds. After long time incubation, the results demonstrated that 3D conditions increased reprogramming efficiency with high levels of pluripotency in comparison with the conventional 2D method. Another reprogramming method, nuclear transfer (NT), was also detected with high improved efficiency when using the MEFs from 3D as nuclear donor. In addition, reprogramming inhibitors namely $p 21$ and B-cell translocation gene 2 (Btg2), were suppressed during cultivation in 3D collagen scaffolds.

Our first experiment is to investigate the effects of 3D collagen scaffolds for fibroblast cell viability. MEFs were respectively seeded on 2D cell plates and in 3D collagen scaffolds at the same time. After 5 days culture, the cells in $3 \mathrm{D}$ collagen scaffolds were characterized by SEM which showed different patterns from $2 \mathrm{D}$ (Fig. 1A). AlarmBlue ${ }^{\circledR}$ cell viability assay showed that MEFs cultured in 3D collagen scaffolds had a better viability than those cultured in 2D (Fig. 1B). To further verify the role of cells culture in different conditions, MEFs were collected for qPCR analysis after 5 days culture. MEFs cultured in 3D collagen scaffolds showed lower expression of senescence markers, p21 and Btg2, compared to the MEFs cultured in 2D (Fig. 1C). The down-regulation of $p 21$ and Btg2 might promote the metabolism of G1 phase cells and speed up the cell multiplication (Tirone, 2001). Moreover, p21 and Btg 2 have been also known as two reprogramming inhibitors, the down-regulation of them might boost reprogramming (Bao et al., 2015).

To study the effect of 3D collagen scaffolds on reprogramming, therefore, we used two approaches to reprogram MEFs into iPSCs (Fig. 1D). Firstly, MEFs were grown in 3D collagen scaffolds. Four days later, one part of MEFs was directly reprogrammed in 3D collagen scaffolds (3D), another part of MEFs was digested and seeded on $2 \mathrm{D}$ cell plates then reprogrammed (3D/2D). Consequently, the group of $3 D$ and $3 D / 2 D$ showed a higher efficiency and higher colony numbers compared to those in 2D conditions (Fig. 1E and $1 F$ ). When the iPSCs were re-seeded in the 3D scaffolds, the colony formed a grape-like cluster within the pores of $3 \mathrm{D}$ collagen scaffolds (Fig. 2A). The limited space on 2D plates inhibited growth of the mouse iPSCs due to the cell-cell connections on the third day whereas the cells grew consistently in 3D scaffolds for at least 6 days (Fig. 2B). The mRNA expression results showed that Oct4, Zfp42, Gata4, Sox2 and KIf4 were significantly up-regulated except for $c-M y c$ in mouse iPSCs in 3D collagen scaffolds than those cultured on $2 \mathrm{D}$ cell plates (Fig. $2 \mathrm{C}$ ). These results indicated that 3D collagen scaffolds could enhance cell proliferation and stemness of mouse iPSCs. The higher pluripotency demonstrates its future developmental ability (Jiang et al., 2011). Down-regulation of $c-M y c$ will reduce risks of tumor formation in grafting experiments (Baudino et al., 2002).

To further confirm the role of $3 \mathrm{D}$ collagen scaffolds in reprogramming, pig iPSCs, commonly difficult to silent their exogenous activation, were derived in $3 \mathrm{D}$ conditions from PEFs. PEFs were directly reprogrammed in $3 D$ collagen scaffolds and typical colonies can be observed by SEM (Fig. S1A). Pig iPSCs were cultured in 3D conditions and longer lasting cell viability was observed in $3 \mathrm{D}$ compared to $2 \mathrm{D}$ conditions (Fig. S1B). The core problem of pig iPSCs is the persistent expression of transgenic genes (Petkov et al., 2015). Our result suggested that the expressions of exogenous, Oct4 and c-Myc, were down-regulated compared to 2D condition (Figure S1C). The endogenous genes of stemness, Oct4, Sox2, Rex1 and Nanog, were up-regulated when cells were reprogrammed in 3D collagen scaffolds (Figure S1D). The down-regulation of the exogenous genes and up-regulation of the endogenous genes (Fig. S1C and S1D) may bring 

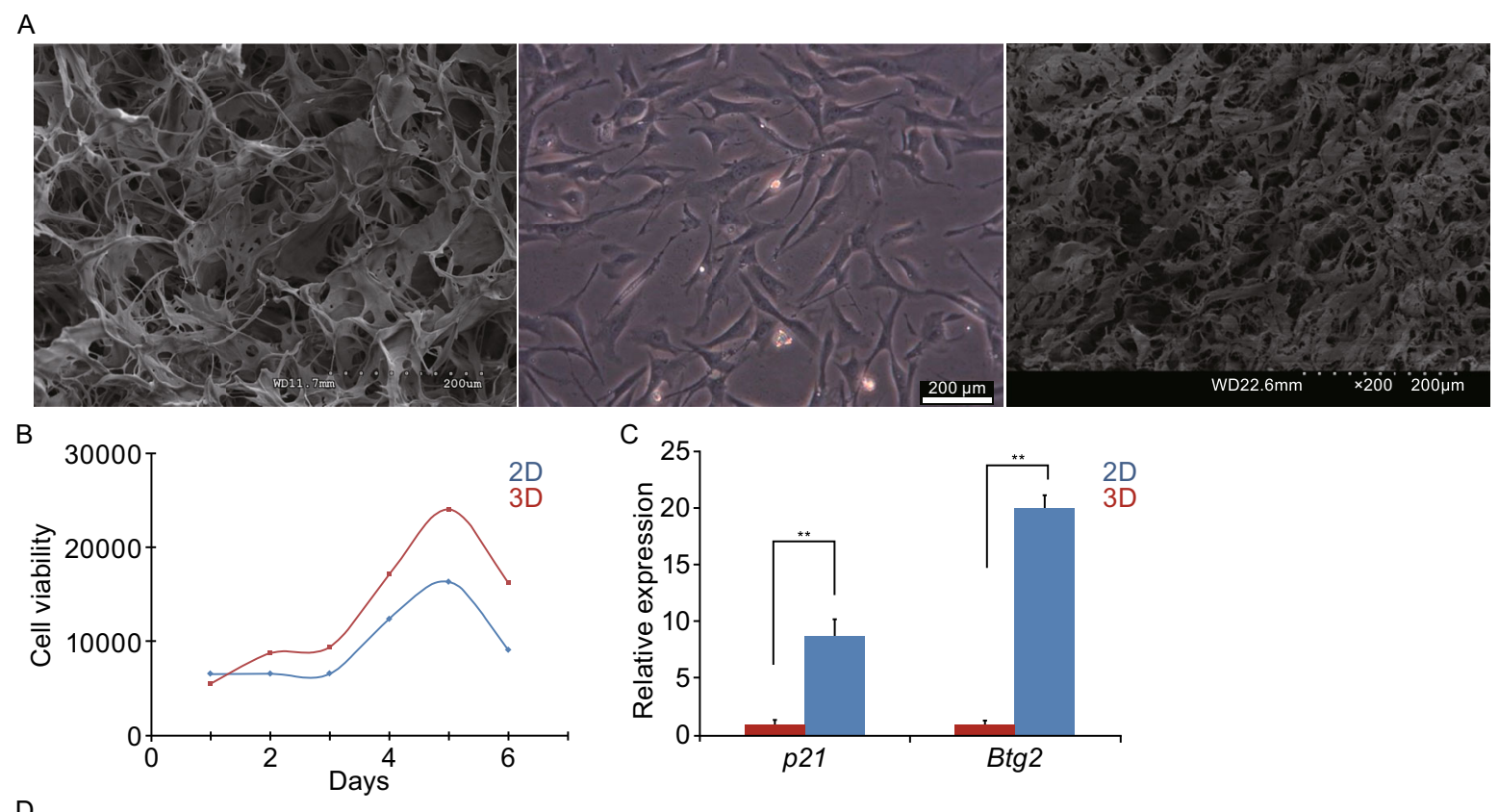

D
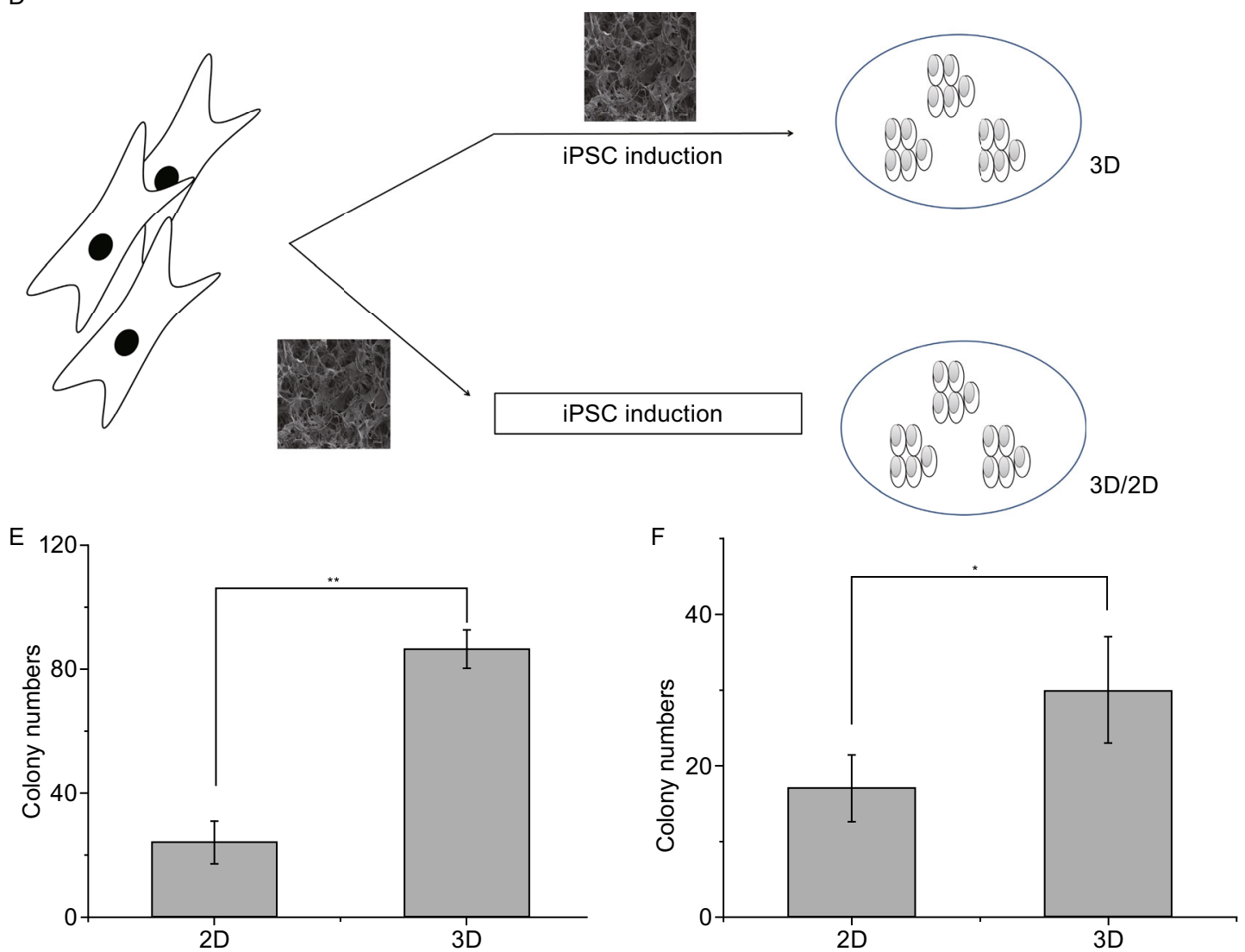

Figure 1. 3D scaffolds improved MEFs growth and mouse iPSC generation. (A) SEM images of 3D collagen scaffolds (left) and MEFs in 3D collagen scaffolds (right) with 2D control monolayer MEFs (middle). Scale bars $=200 \mu \mathrm{m}$. (B) Cell viability analysis for MEFs in 2D and 3D. Y axis number is the value of fluorescence (540/590). (C) qPCR analysis of senescence genes ( $p 21$ and Btg2) expression. MEFs in 3D had lower expression of the genes compared to 2D (mean \pm s.e.m.; one-way ANOVA, Bonferroni comparison test: $\left.{ }^{*} P<0.001\right)$. (D) The scheme of mouse iPSC generation in 3D scaffolds. Up, MEFs were directly induced in 3D scaffolds. Bottom, MEFs were induced on 2D plates after 3D culture. (E) The statistical diagram of colony numbers of 3D and 2D (mean \pm s.e. m.; one-way ANOVA, Bonferroni comparison test: $\left.{ }^{* *} P<0.01\right)$. (F) The statistical diagram of colony numbers of $3 \mathrm{D} / 2 \mathrm{D}$ and $2 \mathrm{D}$ (mean \pm s.e.m.; one-way ANOVA, Bonferroni comparison test: * $P<0.05)$. 

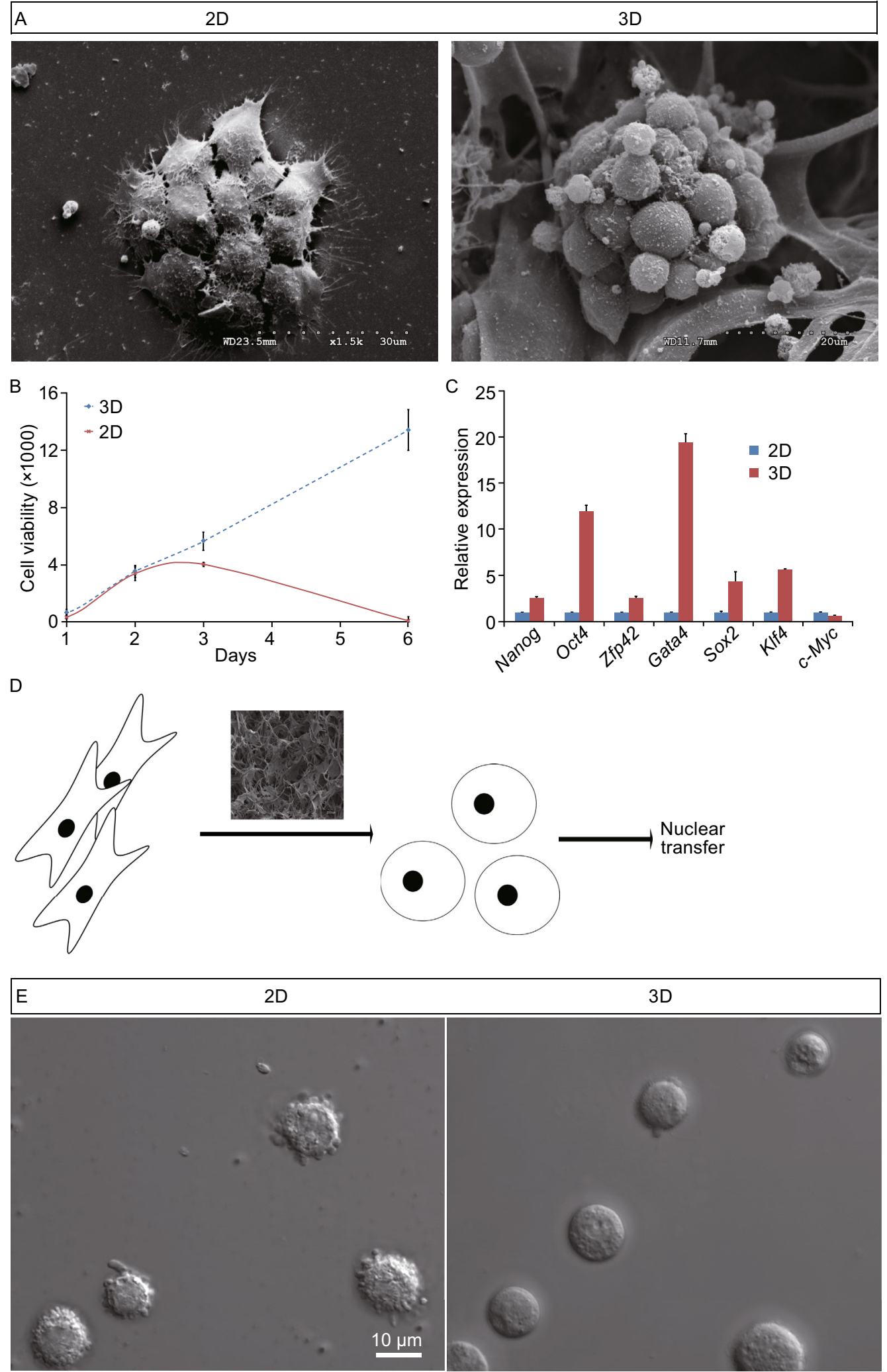

Figure 2. 3D scaffolds used for mouse iPSC and NT. (A) Scanning images of mouse iPSCs on 2D plates (left) and in 3D scaffolds (right). (B) Cell viability analysis of mouse iPSCs on 2D plates and in 3D scaffolds. Y axis number is the value of fluorescence (540/ 590). (C) qPCR analysis of pluripotent gene expression for mouse iPSCs on 2D plates and in 3D scaffolds (mean \pm s.e.m.). (D) The scheme of NT with donors of MEFs in 3D scaffolds. (E) The single cell status of MEFs on 2D plates and in 3D scaffolds. 
forward a new approach for reprogramming without Doxycycline (Fujishiro et al., 2013).

Additionally, NT is part of the classic reprogramming methods. NT efficiency is affected by the conditions of the donor cells (Blelloch et al., 2006). In order to understand the influence of 3D culture on NT, MEFs cultured in 3D collagen scaffolds were digested into single cells and served as donors for NT (Fig. 2D). The results were compared and they performed significantly different from $2 \mathrm{D}$ group of embryo developments, which included 2-cell, 4-cell, morulae and blastocyst (Table S1). The single cells digested from 3D appeared smooth with no filopodia visible around the edges (Fig. 2E). This suggests that filopodia may not play a role in the proliferation in $3 D$ system, which is different from the present 2D studies (Twarock et al., 2010; Arjonen et al., 2011). During a revision of this work, an independent study (Caiazzo et al., 2016) also reported an iPSCs generation in 3D microenvironments, which supports part of our work. However, we used 3D porous scaffolds whereas they have developed a 3D encapsulation culture.

In summary, the relationship between 3D conditions and stem cell has attracted considerable attentions ( $\mathrm{Gu}$ et al., 2015). Our study may provide a novel and useful avenue for stem cell research.

\section{FOOTNOTES}

This work was supported by grants from National Basic Research Program of China (Grant NO .2011CB965001 to L.L.) and Strategic Priority Research Program of Chinese Academy of Sciences (Grant No. XDA01020101 to Q.Z.).

Jie Hao, Qi Zhou, Qi Gu, He Zhu, Lei Chen, Ling Shuai, Jinhui Fang, Jun Wu, Lei Liu, Wei Li, and Jianwu Dai declare that they have no conflict of interest.

All studies were carried out in accordance with the Guidelines of the Care and Use of Laboratory Animals established by Beijing Association for Laboratory Animal Science. All experiments were approved by the Institute of Zoology, Chinese Academy of Sciences.

Qi Gu ${ }^{1,2}, \mathrm{He} \mathrm{Zhu}^{1}$, Lei Chen ${ }^{3}$, Ling Shuai ${ }^{1}$, Jinhui Fang ${ }^{1,4}$, Jun $\mathrm{Wu}^{1}$, Lei Liu ${ }^{1}$, Wei $\mathrm{Li}^{1}$, Jianwu Dai ${ }^{3}$, Jie Hao ${ }^{1 凶}$, Qi Zhou ${ }^{1 凶}$

1 State Key Laboratory of Stem Cell and Reproductive Biology, Institute of Zoology, Chinese Academy of Sciences, Beijing 100101, China

2 AIIM Facility, ARC Centre of Excellence for Electromaterials Science, Intelligent Polymer Research Institute, University of Wollongong, Innovation Campus, Squires Way, Fairy Meadow, NSW 2519, Australia

${ }^{3}$ State Key Laboratory of Molecular Developmental Biology, Institute of Genetics and Developmental Biology, Chinese Academy of Sciences, Beijing 100190, China

${ }^{4}$ College of Life Science, Northeast Agricultural University, Harbin 150030, China

$\bowtie$ Correspondence: haojie@ioz.ac.cn (J. Hao), zhouqi@ioz.ac.cn (Q. Zhou)

\section{OPEN ACCESS}

This article is distributed under the terms of the Creative Commons Attribution 4.0 International License (http://creativecommons.org/ licenses/by/4.0/), which permits unrestricted use, distribution, and reproduction in any medium, provided you give appropriate credit to the original author(s) and the source, provide a link to the Creative Commons license, and indicate if changes were made.

\section{REFERENCES}

Arjonen A, Kaukonen R, Ivaska J (2011) Filopodia and adhesion in cancer cell motility. Cell Adhes Migr 5:421-430

Bao X, Wu H, Zhu X, Guo X, Hutchins AP, Luo Z, Song H, Chen Y, Lai K, Yin M et al (2015) The p53-induced lincRNA-p21 derails somatic cell reprogramming by sustaining $\mathrm{H} 3 \mathrm{~K} 9 \mathrm{me} 3$ and $\mathrm{CpG}$ methylation at pluripotency gene promoters. Cell Res 25:80-92

Baudino TA, McKay C, Pendeville-Samain H, Nilsson JA, Maclean $\mathrm{KH}$, White EL, Davis AC, Ihle JN, Cleveland JL (2002) C-Myc is essential for vasculogenesis and angiogenesis during development and tumor progression. Genes Dev 16:2530-2543

Blelloch R, Wang Z, Meissner A, Pollard S, Smith A, Jaenisch R (2006) Reprogramming efficiency following somatic cell nuclear transfer is influenced by the differentiation and methylation state of the donor nucleus. Stem Cells 24:2007-2013

Caiazzo M, Okawa Y, Ranga A, Piersigilli A, Tabata Y, Lutolf MP (2016) Defined three-dimensional microenvironments boost induction of pluripotency. Nat Mater 15(3):344-352

Di Lullo GA, Sweeney SM, Korkko J, Ala-Kokko L, San Antonio JD (2002) Mapping the ligand-binding sites and disease-associated mutations on the most abundant protein in the human, type I collagen. J Biol Chem 277:4223-4231

Fujishiro SH, Nakano K, Mizukami Y, Azami T, Arai Y, Matsunari H, Ishino R, Nishimura T, Watanabe M, Abe T et al (2013) Generation of naive-like porcine-induced pluripotent stem cells capable of contributing to embryonic and fetal development. Stem Cells Dev 22:473-482

Gu Q, Hao J, Lu Y, Wang L, Wallace GG, Zhou Q (2015) Threedimensional bio-printing. Sci China Life Sci 58:411-419

Gu Q, Tomaskovic-Crook E, Lozano R, Chen Y, Kapsa RM, Zhou Q, Wallace GG, Crook JM (2016) Functional 3D neural mini-tissues from printed gel-based bioink and human neural stem cells. Adv Healthcare Mater 5(12):1429-1438

Jiang J, Ding G, Lin J, Zhang M, Shi L, Lv W, Yang H, Xiao H, Pei G, Li Y (2011) Different developmental potential of pluripotent stem cells generated by different reprogramming strategies. J Mol Cell Biol 3:197-199

Keung AJ, Kumar S, Schaffer DV (2010) Presentation counts: microenvironmental regulation of stem cells by biophysical and material cues. Annu Rev Cell Dev Biol 26:533-556

Petkov S, Glage S, Nowak-Imialek M, Niemann H (2015) Long-term culture of porcine induced pluripotent stem-like cells under feeder-free conditions in the presence of histone deacetylase inhibitors. Stem Cells Dev 1(6):e23 
Song T, Zhao X, Sun H, Li X, Lin N, Ding L, Dai J, Hu Y (2015) Regeneration of uterine horns in rats using collagen scaffolds loaded with human embryonic stem cell-derived endometriumlike cells. Tissue Eng Part A 21:353-361

Tirone F (2001) The gene PC3(TIS21/BTG2), prototype member of the PC3/BTG/TOB family: regulator in control of cell growth, differentiation, and DNA repair? J Cell Physiol 187:155-165
Twarock S, Tammi MI, Savani RC, Fischer JW (2010) Hyaluronan stabilizes focal adhesions, filopodia, and the proliferative phenotype in esophageal squamous carcinoma cells. J Biol Chem 285:23276-23284

Qi Gu and He Zhu have contributed equally to this work.

Electronic supplementary material The online version of this article (doi:10.1007/s13238-016-0321-2) contains supplementary material, which is available to authorized users. 